Article

\title{
Selective and Efficient Generation of ortho-Brominated para-Substituted Phenols in ACS-Grade Methanol
}

\author{
David Georgiev, Bartholomeus W. H. Saes, Heather J. Johnston, Sarah K. Boys, Alan Healy \\ and Alison N. Hulme*
}

Received: 28 September 2015; Accepted: 7 January 2016; Published: 13 January 2016

Academic Editor: Kerry Gilmore

EaStCHEM School of Chemistry, The University of Edinburgh, David Brewster Road, Edinburgh EH9 3FJ, UK; D.Georgiev@sms.ed.ac.uk (D.G.); bartsaes@live.nl (B.W.H.S.); hjohnst2@exseed.ed.ac.uk (H.J.J.);

boys.sarah@gmail.com (S.K.B.); alan.healy@yale.edu (A.H.)

* Correspondence: Alison.Hulme@ed.ac.uk; Tel.: +44-131-650-4711; Fax: +44-131-650-4743

\begin{abstract}
The mono ortho-bromination of phenolic building blocks by NBS has been achieved in short reaction times (15-20 $\mathrm{min}$ ) using ACS-grade methanol as a solvent. The reactions can be conducted on phenol, naphthol and biphenol substrates, giving yields of $>86 \%$ on gram scale. Excellent selectivity for the desired mono ortho-brominated products is achieved in the presence of $10 \mathrm{~mol} \%$ para- $\mathrm{TsOH}$, and the reaction is shown to be tolerant of a range of substituents, including $\mathrm{CH}_{3}, \mathrm{~F}$, and $\mathrm{NHBoc}$.
\end{abstract}

Keywords: ortho-bromination; ACS-grade methanol; NBS

\section{Introduction}

Brominated phenols and their derivatives constitute important building blocks for a range of synthetic targets (Figure 1), with recent examples of pharmaceutical interest including: (i) synthesis of aryl 1-indanylketone inhibitors of the human peptidyl prolyl cis/trans isomerase Pin1, such as 1, using a domino coupling reaction starting from the methylated derivative of 2-bromo-4-fluorophenol 2 [1]; (ii) synthesis of alkynylphenoxyacetic acid CRTH2 (DP2) receptor antagonists, such as library members 3, by Sonogashira coupling to the tert-butyl protected phenoxyacetic acid derivative of 3-bromo-[1,1'-biphenyl]-4-ol 4 [2]; and (iii) synthesis of the cytotoxic peptidic marine natural product bisebromoamide 5 [3], through modification and peptide coupling of brominated D-tyrosine derivatives such as $6[4,5]$.
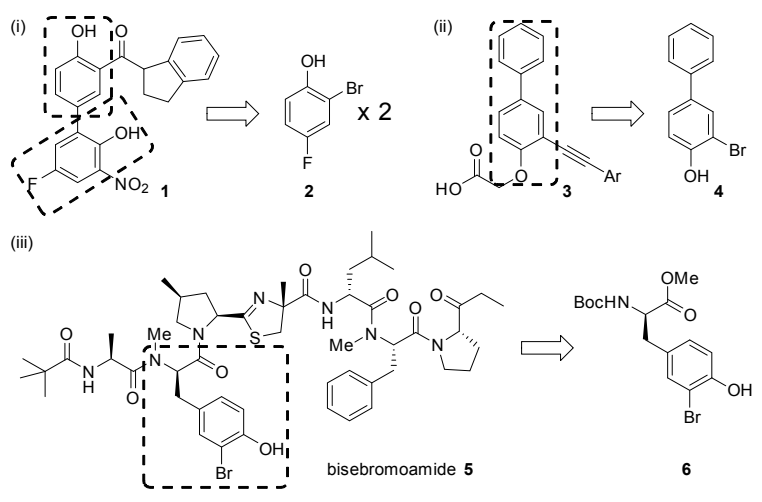

Figure 1. Recent applications of mono ortho-brominated phenols to the synthesis of biologically active targets. 
As part of our own synthetic efforts directed towards the efficient solid-phase synthesis of bisebromoamide 5 [6,7], gram-scale syntheses of each of the component building blocks was required [8,9]. For the bromotyrosine derivative a number of recently published methods for the $\mathrm{N}$-bromosuccinimide (NBS) promoted mono-ortho-bromination reaction were surveyed with a view to applying them to the amino acid derivative Boc-D-Tyr-OMe $(\mathbf{7} \rightarrow \mathbf{6}$, Scheme 1$)$. Of particular concern was minimization of over-bromination which results in the dibromo derivative 8 , removal of which by chromatography greatly reduced synthetic efficiency.

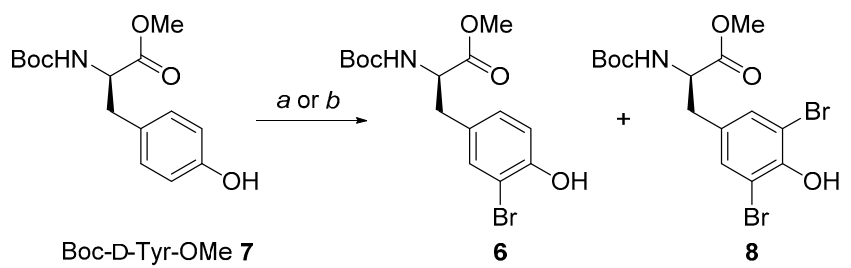

Scheme 1. Building block synthesis for the marine natural product bisebromoamide 5. Reagents and Conditions: (a) NBS (100 mol \%), In(OTf) 3 (10 mol \%), MeCN, rt, $10 \mathrm{~min} ;$ (b) NBS (100 mol \%), pTsOH (10 mol \%), EtOAc, rt, $30 \mathrm{~h}$.

Initial attempts to brominate Boc-D-Tyr-OMe 7 focused on indium(III) triflate catalysis of the reaction of NBS in acetonitrile [10]. However, the major product of the reaction was found to be the dibrominated material 8 , even when the reaction was conducted in the dark and at reduced temperature $\left(0^{\circ} \mathrm{C}\right)$. The Chhattise group have demonstrated rapid $(<10 \mathrm{~min})$ photochemical bromination of aromatic compounds (including para-substituted phenols) under UV-vis irradiation at ambient temperatures [11]. Reaction of Boc-D-Tyr-OMe 7 with one equivalent of NBS in ethyl acetate under UV-vis irradiation (365 nm; $6 \mathrm{~W}$, F6T5/BL lamp) resulted in rapid consumption of the starting material, but again gave predominantly the undesired dibrominated product 8 .

In an attempt to minimize the dibromination reaction, attention then shifted to the $p$-toluene-sulfonic acid ( $p \mathrm{TsOH})$-mediated NBS bromination reaction [12,13]. Leykajarakul and co-workers have proposed a mechanism for this reaction in which the $p \mathrm{TsOH}$ conjugates to the phenolic alcohol directing bromination to the para position, or in the case of para-substituted phenols to give selective mono ortho-bromination $[11,13,14]$. When Boc-D-Tyr-OMe 7 was reacted with NBS in the presence of $p \mathrm{TsOH}(10 \mathrm{~mol} \%)$ in ethyl acetate a moderate yield (58\%) of the mono-brominated material 6 was obtained after $30 \mathrm{~h}$ reaction time. UV-vis irradiation (365 nm; $6 \mathrm{~W}, \mathrm{~F} 6 \mathrm{~T} 5 / \mathrm{BL}$ lamp) of this reaction gave full conversion to the desired monobrominated product 6 in times ranging from $40 \mathrm{~min}$ to $4 \mathrm{~h}$, depending on the reaction scale and light source used. However, it was suspected that the extended reaction times also led to UV-mediated debromination of the desired product, complicating the reaction still further. For these reasons, reproducible reaction conditions which were readily amenable to scale-up were sought. Flow chemistry, and in particular photochemical flow chemistry with its short path lengths allowing efficient irradiation, readily controlled residence times and scaleability [15-19], was initially considered as a potential solution.

\section{Results and Discussion}

\subsection{Investigation of the Mono ortho-Bromination Flow Reaction}

Investigation of the mono ortho-bromination reaction by NBS in the presence of $p \mathrm{TsOH}(10 \mathrm{~mol} \%)$ under flow conditions was carried out using a UV photoreactor (365-366 nm; $125 \mathrm{~W}, \mathrm{Hg}$ lamp) based on those reported by the Booker-Milburn and Seeberger groups [16-19]. The initial batch reactions had been conducted in ethyl acetate, but NBS shows only limited solubility in this solvent making it incompatible with a flow modality for the reaction. For this reason the flow reaction was explored in both acetonitrile and methanol; two solvents which are commonly used in bromination reactions on other classes of substrates. Although higher concentrations of NBS could be achieved in acetonitrile 
than in methanol (0.5 M and 0.1 M respectively) which would lead to increased production rates in flow, the reactions in acetonitrile were noticeably slower with longer residence times required in the UV photoreactor to achieve full conversion. Indeed, across a range of substrates the observed rates of reaction in methanol were so fast that it led us to suspect that exposure to the UV light source was not required. This was confirmed by reactions conducted under standard flow conditions but in the absence of UV light (the so-called "dark reaction"), in which reactive substrates showed full conversion to the mono ortho-brominated products in methanol, but only minimal conversion in acetonitrile. These observations led us to look more closely at the NBS-mediated mono ortho-bromination reaction in methanol.

\subsection{Investigation of Batch Reaction Conditions Using ACS-Grade Methanol}

There are very few reports of ortho-bromination reactions of phenols conducted in methanol; and those which have been reported to give mono selectivity rely on blocking of the second ortho position to achieve this [14]. However, a similar dramatic rate acceleration for the NBS-mediated bromination of the polyalkylated aromatic durene in the presence of $p \mathrm{TsOH}$ has been reported when this reaction was conducted in methanol rather than acetonitrile, or ethyl acetate [20]. The facile chlorination of aromatic substrates in water at $40^{\circ} \mathrm{C}$ using a combination of $\mathrm{NCS} / \mathrm{NaCl}$ reagents in the presence of $p \mathrm{TsOH}$ has also been reported [21,22]. It has been proposed that under these polar protic conditions the $p \mathrm{TsOH}$ accelerates the reaction through protonation of the $N$-halosuccinimide to provide a more reactive electrophile $[20,21]$.

In investigating the selective mono ortho-bromination reaction by NBS in the presence of $p$ TsOH in methanol, the reaction of $p$-cresol 9 (Table 1) was used as a test substrate, as it was both readily available and the reaction to give the desired mono ortho-brominated product $\mathbf{1 0}$ (and the undesired dibrominated product 11) could be easily followed by HPLC (SI Figure S1). The p-cresol 9 was premixed with $p$ TsOH prior to addition of NBS as this has been shown to give better selectivity for mono ortho-bromination [12]. HPLC analysis of samples taken directly from the reaction mixture at timed intervals rapidly established that the batch reaction of 9 in methanol reached completion in under $5 \mathrm{~min}$, even in the absence of light. When the reaction was carried out with NBS addition in a single portion at the beginning of the reaction, bromination of $p$-cresol 9 gave conversion to 9:10:11 in a ratio of 6:87:7 (Table 1 , entry 1 ).

Table 1. Optimization of batch conditions for the mono ortho-bromination reaction.

\begin{tabular}{|c|c|c|c|c|c|c|}
\hline \multirow{2}{*}{ Entry } & \multirow{2}{*}{ NBS (mol \%) } & \multirow{2}{*}{$p \mathrm{TsOH}(\mathrm{mol} \%)$} & \multicolumn{3}{|c|}{ Ratio (Relative \% at $285 \mathrm{~nm}$ ) } & \multirow{2}{*}{$\begin{array}{c}\text { Isolated Yield } \\
10(\%)\end{array}$} \\
\hline & & & 9 & 10 & 11 & \\
\hline $1^{\mathrm{a}, \mathrm{b}}$ & 100 & 10 & 6 & 87 & 7 & - \\
\hline $2^{b}$ & 100 & 10 & 5 & 93 & 2 & - \\
\hline 3 & 100 & 10 & 3 & 94 & 3 & $92 \%$ \\
\hline 4 & 110 & 10 & 0 & 88 & 12 & $86 \%$ \\
\hline 5 & 100 & 0 & 15 & 77 & 8 & $74 \%$ \\
\hline 6 & 100 & 20 & 3 & 93 & 4 & $90 \%$ \\
\hline
\end{tabular}

${ }^{a}$ NBS added as a single portion at the start of the reaction and then reaction stirred for $25 \mathrm{~min} ;{ }^{\mathrm{b}}$ Reaction conducted in dry methanol.

When the bulk concentration of NBS was reduced through its controlled addition in solution by cannula, an overall increase in conversion to the desired mono ortho-brominated product 10 was observed (Table 1, entry 2). The reaction was found to be tolerant of moisture (Table 1, entries 2 and 3); when the reaction was conducted in ACS-grade methanol, under ambient conditions (air and room 
temperature) an excellent isolated yield of $\mathbf{1 0}$ was achieved and no significant change in the ratio of products was observed. Selectivity for the desired mono ortho-brominated product was shown to depend heavily on the number of equivalents of NBS used (Table 1, entries 3 and 4), but also to be influenced by the presence of $p \mathrm{TsOH}$ (Table 1, entries 5 and 6) as previously reported [12,13].

Optimum conditions involved pre-mixing the substrate and $p \mathrm{TsOH}(10 \mathrm{~mol} \%)$ in a minimal amount of ACS-grade methanol and controlled addition of a solution of NBS (100 mol \%) as a $0.1 \mathrm{M}$ solution in methanol over $20 \mathrm{~min}$, then stirring for a further $5 \mathrm{~min}$, at ambient temperature $\left(\sim 20^{\circ} \mathrm{C}\right)$ under air; giving a ratio of 9:10:11 of 3:94:3 and an isolated yield of 9 of $92 \%$. These conditions were then successfully applied to a range of substrates on gram scale as shown in Table 2.

Table 2. Gram scale mono ortho-bromination reaction in ACS-grade methanol.

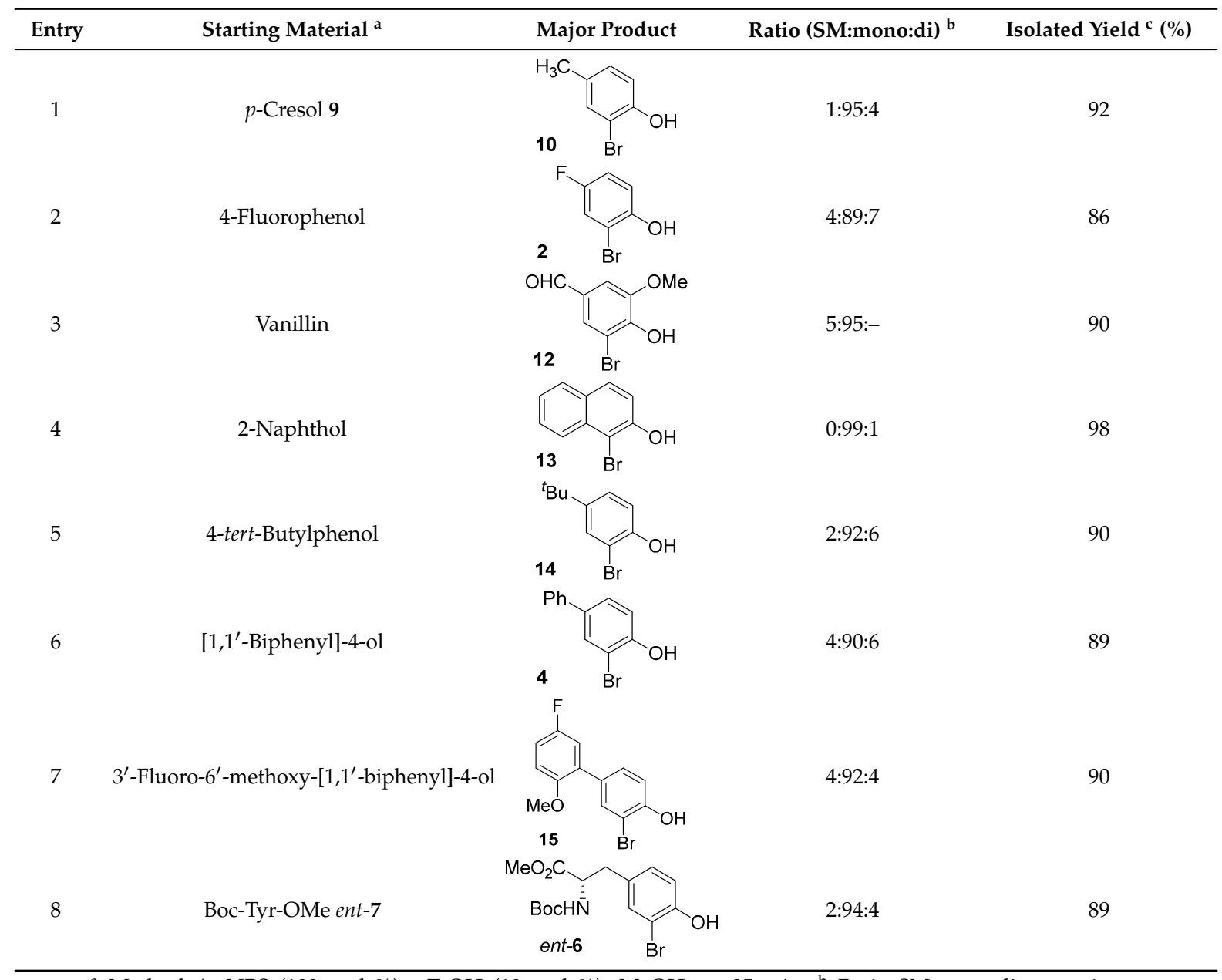

${ }^{a}$ Method A: NBS (100 mol \%), $p$ TsOH (10 mol \%), MeOH, rt, 25 min; ${ }^{b}$ Ratio SM:mono:di = starting material:mono ortho-brominated:di ortho-brominated; ${ }^{\mathrm{c}}$ Major product.

Phenol derivatives with alkyl and aryl para-substituents (entries 1, 5-7, Table 2) and Boc-Tyr-OMe (ent-7, entry 8, Table 2) were shown to give high selectivity for the mono ortho-brominated products under these conditions, facilitating separation to give excellent yields of the major product. Vanillin and 2-naphthol (entries 3 and 4, Table 2), both known to react readily to bromination, also gave excellent conversion. Electron withdrawing para-substituents (entry 2, Table 2, and $p-\mathrm{CF}_{3}$ not shown) generally induced poorer selectivity for the mono ortho-brominated products resulting in somewhat reduced isolated yields of the major products. 


\section{Experimental Section}

\subsection{General Information}

ACS-grade methanol was obtained from Fischer Scientific and was used without further purification or drying; all other chemicals were used as obtained from the supplier unless otherwise stated. ${ }^{1} \mathrm{H}-,{ }^{13} \mathrm{C}$ - and ${ }^{19} \mathrm{~F}-\mathrm{NMR}$ spectra were obtained on an AVA 500 or a PRO 500 instrument (Bruker, Coventry, UK) using TMS as a reference and residual solvent as an internal standard. The data are presented as follows: chemical shift (in ppm on the $\delta$ scale relative to $\delta_{\mathrm{TMS}}=0$ ), integration, multiplicity, coupling constant and interpretation. Electron ionisation (EI) mass spectra were obtained on a MS50TC mass spectrometer (Kratos, Manchester, UK). Analytical Reverse Phase HPLC (RP-HPLC) was conducted on a Waters ${ }^{\circledR} 600(100 \mu \mathrm{L})$ system (Waters, Elstree, UK) using a 717plus autosampler and 996 PDA detector (190 to $800 \mathrm{~nm}$ ) equipped with a Phenomenex ${ }^{\circledR}$ Luna C18(2) $5 \mu \mathrm{m}$ column (i.d. $4.6 \mathrm{~mm}$, length $300 \mathrm{~mm})$. A binary solvent system was used $\mathrm{A}=$ water $(0.1 \% \mathrm{TFA}), \mathrm{B}=\mathrm{MeCN}$ $(0.1 \% \mathrm{TFA})$ at a flow rate of $1.00 \mathrm{~mL} \cdot \mathrm{min}^{-1}$; and the column was maintained at $30 \pm 1^{\circ} \mathrm{C}$. The elution program was a linear gradient from $0 \mathrm{~min}(95 \mathrm{~A}: 5 \mathrm{~B})$ to $30 \mathrm{~min}(5 \mathrm{~A}: 95 \mathrm{~B})$, isocratic from $30 \mathrm{~min}$ to $35 \mathrm{~min}$ (5A:95B), before recovery of the initial conditions over $5 \mathrm{~min}$ and equilibration over $10 \mathrm{~min}$, giving a total run time of $50 \mathrm{~min}$. Melting points were determined on an Electrothermal Melting Point apparatus (Gallenkamp, Loughborough, UK) and are uncorrected. Optical rotations were performed on a POLAAR 20 polarimeter (Optical Activity, Ramsey, UK).

\subsection{General Procedure for Batch Reaction Conditions in ACS-Grade Methanol}

A solution of the starting material ( $10 \mathrm{mmol})$ and $p \mathrm{TsOH}(10 \mathrm{~mol} \%)$ in $\mathrm{MeOH}(1.0 \mathrm{~mL}$ per mmol starting material) was stirred for $10 \mathrm{~min}$, then a solution of NBS $\left(100 \mathrm{~mol} \%\right.$; recrystallized from $\left.\mathrm{H}_{2} \mathrm{O}\right)$ in $\mathrm{MeOH}(0.1 \mathrm{M})$ was added dropwise over $20 \mathrm{~min}$ from a foiled reaction flask. The reaction mixture was stirred for a further $5 \mathrm{~min}$ and then concentrated in vacuo. The resultant residue was purified using column chromatography $\left(\mathrm{CH}_{2} \mathrm{Cl}_{2}\right.$, or $1 \% \mathrm{MeOH}$ in $\left.\mathrm{CH}_{2} \mathrm{Cl}_{2}\right)$.

\subsection{Characterization of Products}

2-Bromo-4-methylphenol (10) [23]: $10.1 \mathrm{mmol} ; 1.73 \mathrm{~g}$ (92\%); $\mathrm{Rt}=24.3 \mathrm{~min} ;{ }^{1} \mathrm{H}-\mathrm{NMR} \delta\left(500 \mathrm{MHz}, \mathrm{CDCl}_{3}\right.$ ) $7.30(1 \mathrm{H}, \mathrm{br} \mathrm{s}, \operatorname{ArH}), 7.04(1 \mathrm{H}, \mathrm{br} \mathrm{d}, J=8.2 \mathrm{~Hz}, \operatorname{ArH}), 6.94(1 \mathrm{H}, \mathrm{d}, J=8.2 \mathrm{~Hz}, \operatorname{ArH}), 5.43(1 \mathrm{H}, \mathrm{s}, \mathrm{OH}), 2.30$ $\left(3 \mathrm{H}, \mathrm{s}, \mathrm{OCH}_{3}\right) ;{ }^{13} \mathrm{C}-\mathrm{NMR} \delta\left(126 \mathrm{MHz}, \mathrm{CDCl}_{3}\right) 150.0(\mathrm{C}), 132.2(\mathrm{CH}), 131.4(\mathrm{C}), 129.8(\mathrm{CH}), 115.8(\mathrm{CH})$, 109.9 (C), $20.2\left(\mathrm{CH}_{3}\right) ; m / z$ (EI) $188\left({ }^{81} \mathrm{BrM}^{+}, 52 \%\right), 186\left({ }^{79} \mathrm{BrM}^{+}, 54\right), 107$ (100), 77 (35).

2,6-Dibromo-4-methylphenol (11) [24]: Rt = $27.6 \mathrm{~min} ; \mathrm{mp} 47-49{ }^{\circ} \mathrm{C}$, lit [24] 49-51 ${ }^{\circ} \mathrm{C} ;{ }^{1} \mathrm{H}-\mathrm{NMR} \delta(500 \mathrm{MHz}$, $\left.\mathrm{CDCl}_{3}\right) 7.26(2 \mathrm{H}, \mathrm{br} \mathrm{s}, \mathrm{ArH}), 5.70(1 \mathrm{H}, \mathrm{s}, \mathrm{OH}), 2.25\left(3 \mathrm{H}, \mathrm{s}, \mathrm{OCH}_{3}\right) ;{ }^{13} \mathrm{C}-\mathrm{NMR} \delta\left(126 \mathrm{MHz}, \mathrm{CDCl}_{3}\right) 147.1$ (C), $132.4\left(2 \times \mathrm{CH}\right.$ and C), $109.4(2 \times \mathrm{C}), 20.0\left(\mathrm{CH}_{3}\right) ; m / z$ (EI) $268\left({ }^{81} \mathrm{Br}^{81} \mathrm{BrM}^{+}, 46 \%\right), 266\left({ }^{81} \mathrm{Br}^{79} \mathrm{BrM}^{+}\right.$, 100), $264\left({ }^{79} \mathrm{Br}^{79} \mathrm{BrM}^{+}, 48\right), 187$ (39),185 (41).

2-Bromo-4-fluorophenol (2) [23]: $10.3 \mathrm{mmol} ; 1.70 \mathrm{~g}(86 \%) ; \mathrm{Rt}=23.1 \mathrm{~min} ;{ }^{1} \mathrm{H}-\mathrm{NMR} \delta\left(500 \mathrm{MHz}, \mathrm{CDCl}_{3}\right.$ ) $7.21(1 \mathrm{H}$, ddd, $J=7.7,2.5$ and $0.7 \mathrm{~Hz} \mathrm{ArH}), 7.00-6.93(2 \mathrm{H}, \mathrm{m}, 2 \times \mathrm{ArH}), 5.32(1 \mathrm{H}, \mathrm{s}, \mathrm{OH}) ;{ }^{13} \mathrm{C}-\mathrm{NMR} \delta$ $\left(126 \mathrm{MHz} \mathrm{CDCl}_{3}\right) 156.4\left(\mathrm{~d},{ }^{1} J_{\mathrm{CF}}=242 \mathrm{~Hz}, \mathrm{C}\right), 148.9\left(\mathrm{~d},{ }^{4} J_{\mathrm{CF}}=3 \mathrm{~Hz}, \mathrm{CH}\right), 118.7\left(\mathrm{~d},{ }^{2} J_{\mathrm{CF}}=26 \mathrm{~Hz}, \mathrm{C}\right)$, $116.3\left(\mathrm{~d},{ }^{3} J_{\mathrm{CF}}=8 \mathrm{~Hz}, \mathrm{CH}\right), 116.0\left(\mathrm{~d},{ }^{2} J_{\mathrm{CF}}=23 \mathrm{~Hz}, \mathrm{CH}\right), 109.5\left(\mathrm{~d},{ }^{3} J_{\mathrm{CF}}=10 \mathrm{~Hz}, \mathrm{C}\right) ;{ }^{19} \mathrm{~F}-\mathrm{NMR} \delta(471 \mathrm{MHz}$, $\left.\mathrm{CDCl}_{3}\right)-121.97(\mathrm{td}, J=7.7$ and $5.4 \mathrm{~Hz}) ; \mathrm{m} / z(\mathrm{EI}) 192\left({ }^{81} \mathrm{BrM}^{+}, 98 \%\right), 190\left({ }^{79} \mathrm{BrM}^{+}, 100\right), 82(43), 83(27)$.

3-Bromo-4-hydroxy-5-methoxybenzaldehyde (12) [25]: $9.09 \mathrm{mmol} ; 1.89 \mathrm{~g}(90 \%)$; Rt = $21.4 \mathrm{~min}$; $\mathrm{mp}$ 160-162 ${ }^{\circ} \mathrm{C}$, lit [25] $162-164{ }^{\circ} \mathrm{C}, \mathrm{EtOH} ;{ }^{1} \mathrm{H}-\mathrm{NMR} \delta\left(500 \mathrm{MHz}, \mathrm{DMSO}-d_{6}\right) 10.72(1 \mathrm{H}, \mathrm{s}, \mathrm{OH}), 9.79(1 \mathrm{H}$, $\mathrm{s}, \mathrm{CHO}), 7.73(1 \mathrm{H}, \mathrm{d}, J=1.8 \mathrm{~Hz}, \mathrm{ArH}), 7.43(1 \mathrm{H}, \mathrm{d}, J=1.8 \mathrm{~Hz}, \mathrm{ArH}), 3.92\left(3 \mathrm{H}, \mathrm{s}, \mathrm{OCH}_{3}\right) ;{ }^{13} \mathrm{C}-\mathrm{NMR} \delta$ (126 MHz, DMSO-d 6 ) 190.9 (CH), $150.3(\mathrm{C}), 149.1(\mathrm{C}), 129.4(\mathrm{C}), 129.2(\mathrm{CH}), 110.1(\mathrm{CH}), 109.7(\mathrm{C}), 56.8$ $\left(\mathrm{CH}_{3}\right) ; m / z$ (EI) $232\left({ }^{81} \mathrm{BrM}^{+}, 94 \%\right), 231$ (86) $230\left({ }^{79} \mathrm{BrM}^{+}, 100\right), 229$ (95). 
1-Bromo-2-naphthol (13) [23]: $9.05 \mathrm{mmol} ; 1.98 \mathrm{~g}(98 \%) ; \mathrm{Rt}=26.4 \mathrm{~min} ; \mathrm{mp} 80-82{ }^{\circ} \mathrm{C}$, lit [23] 82-83 ${ }^{\circ} \mathrm{C}$; ${ }^{1} \mathrm{H}-\mathrm{NMR} \delta\left(500 \mathrm{MHz}, \mathrm{CDCl}_{3}\right) 8.06(1 \mathrm{H}, \mathrm{br} \mathrm{d}, J=8.4 \mathrm{~Hz}, \mathrm{ArH}), 7.81(1 \mathrm{H}, \mathrm{br} \mathrm{d}, J=8.1 \mathrm{~Hz}, \mathrm{ArH}), 7.77(1 \mathrm{H}$, $\mathrm{d}, J=8.8 \mathrm{~Hz}, \mathrm{ArH}), 7.60(1 \mathrm{H}, \mathrm{ddd}, J=8.4,6.9$ and $1.2 \mathrm{~Hz}, \mathrm{ArH}), 7.42(1 \mathrm{H}, \mathrm{ddd}, J=8.1,6.9 \mathrm{and} 1.0 \mathrm{~Hz}$, $\mathrm{ArH}), 7.30(1 \mathrm{H}, \mathrm{d}, J=8.8 \mathrm{~Hz}, \mathrm{ArH}), 5.94(1 \mathrm{H}, \mathrm{s}, \mathrm{OH}) ;{ }^{13} \mathrm{C}-\mathrm{NMR} \delta\left(126 \mathrm{MHz}, \mathrm{CDCl}_{3}\right) 150.6(\mathrm{C}), 132.3(\mathrm{C})$, $129.7(\mathrm{C}), 129.4(\mathrm{CH}), 128.2(\mathrm{CH}), 127.9(\mathrm{CH}), 125.3(\mathrm{CH}), 124.2(\mathrm{CH}), 117.2(\mathrm{CH}), 106.2(\mathrm{C}) ; \mathrm{m} / z(\mathrm{EI}) 224$ $\left({ }^{81} \mathrm{BrM}^{+}, 98 \%\right), 222\left({ }^{79} \mathrm{BrM}^{+}, 100\right), 115$ (26), 114 (36).

2-Bromo-4-tert-butylphenol (14) [26]: $10.1 \mathrm{mmol} ; 2.07 \mathrm{~g}(90 \%) ; \mathrm{Rt}=27.6 \mathrm{~min} ;{ }^{1} \mathrm{H}-\mathrm{NMR} \delta(500 \mathrm{MHz}$, $\left.\mathrm{CDCl}_{3}\right) 7.48(1 \mathrm{H}, \mathrm{d}, J=2.3 \mathrm{~Hz}, \mathrm{ArH}), 7.27(1 \mathrm{H}, \mathrm{dd}, J=8.5$ and $2.3 \mathrm{~Hz}, \mathrm{ArH}), 6.99(1 \mathrm{H}, \mathrm{d}, J=8.5 \mathrm{~Hz}, \mathrm{ArH})$, $5.39(1 \mathrm{H}, \mathrm{s}, \mathrm{OH}), 1.32\left(9 \mathrm{H}, \mathrm{s}, \mathrm{C}\left(\mathrm{CH}_{3}\right)_{3}\right) ;{ }^{13} \mathrm{C}-\mathrm{NMR} \delta\left(126 \mathrm{MHz}, \mathrm{CDCl}_{3}\right) 149.9(\mathrm{C}), 145.1(\mathrm{C}), 128.8(\mathrm{CH})$, $126.3(\mathrm{CH}), 115.6(\mathrm{CH}), 109.9(\mathrm{C}), 34.2(\mathrm{C}), 31.4\left(3 \times \mathrm{CH}_{3}\right) ; m / z(\mathrm{EI}) 230\left({ }^{81} \mathrm{BrM}^{+}, 76 \%\right), 228\left({ }^{79} \mathrm{BrM}^{+}\right.$, 74), 215 (100), 213 (86), 134 (87).

3-Bromo-[1,1'-biphenyl]-4-ol (4) [27]: $10.0 \mathrm{mmol} ; 2.22 \mathrm{~g}$ (89\%); Rt = $24.2 \mathrm{~min} ; \mathrm{mp} 92-94{ }^{\circ} \mathrm{C}$, lit [27] 94-95 ${ }^{\circ} \mathrm{C} ;{ }^{1} \mathrm{H}-\mathrm{NMR} \delta\left(500 \mathrm{MHz}, \mathrm{CDCl}_{3}\right) 7.70(1 \mathrm{H}, \mathrm{d}, J=2.2 \mathrm{~Hz}), 7.56-7.48(2 \mathrm{H}, \mathrm{m}), 7.46(1 \mathrm{H}, \mathrm{dd}, J=8.4$ and 2.2, Hz), 7.44-7.39 $(2 \mathrm{H}, \mathrm{m}), 7.37-7.29(1 \mathrm{H}, \mathrm{m}), 7.09(1 \mathrm{H}, \mathrm{d}, J=8.4 \mathrm{~Hz}), 5.52(1 \mathrm{H}, \mathrm{s}, \mathrm{OH}) ;{ }^{13} \mathrm{C}-\mathrm{NMR} \delta$ $\left(126 \mathrm{MHz}, \mathrm{CDCl}_{3}\right) 151.7(\mathrm{C}), 139.5(\mathrm{C}), 135.4(\mathrm{C}), 130.5(\mathrm{CH}), 128.9(2 \times \mathrm{CH}), 128.0(\mathrm{CH}), 127.3(\mathrm{CH})$, $126.8(2 \times \mathrm{CH}), 116.3(\mathrm{CH}), 110.7(\mathrm{C}) ; \mathrm{m} / z(\mathrm{EI}) 250\left({ }^{81} \mathrm{BrM}^{+}, 100 \%\right), 248\left({ }^{79} \mathrm{BrM}^{+}, 100\right), 139(38), 86(58)$, 84 (99).

3-Bromo-3'-fluoro-6'-methoxy-[1, $1^{\prime}$-biphenyl]-4-ol (15): $3.49 \mathrm{mmol} ; 0.93 \mathrm{~g}(90 \%) ; \mathrm{Rt}=28.3 \mathrm{~min} ;{ }^{1} \mathrm{H}-\mathrm{NMR}$ $\delta\left(500 \mathrm{MHz}, \mathrm{CDCl}_{3}\right) 7.67(1 \mathrm{H}, \mathrm{d}, J=2.1 \mathrm{~Hz}, \mathrm{ArH}), 7.41(1 \mathrm{H}, \mathrm{dd}, J=8.4$ and $2.1 \mathrm{~Hz}, \mathrm{ArH}), 7.09(1 \mathrm{H}, \mathrm{d}$, $J=8.4 \mathrm{~Hz}, \operatorname{ArH}), 7.03(1 \mathrm{H}, \mathrm{ddd}, J=9.2,3.1$ and $0.7 \mathrm{~Hz}, \operatorname{ArH}), 7.03-6.98(1 \mathrm{H}, \mathrm{m}, \operatorname{ArH}), 6.91(1 \mathrm{H}, \mathrm{ddd}$, $J=8.7,4.5$ and $0.7 \mathrm{~Hz}, \mathrm{ArH}), 5.57(1 \mathrm{H}, \mathrm{s}, \mathrm{OH}), 3.81\left(3 \mathrm{H}, \mathrm{s}, \mathrm{OCH}_{3}\right) ;{ }^{13} \mathrm{C}-\mathrm{NMR} \delta\left(126 \mathrm{MHz}, \mathrm{CDCl}_{3}\right) 157.1$ $\left(\mathrm{d},{ }^{1} J_{\mathrm{CF}}=239 \mathrm{~Hz}, \mathrm{C}\right), 152.5\left(\mathrm{~d},{ }^{4} J_{\mathrm{CF}}=2 \mathrm{~Hz}, \mathrm{C}\right), 151.6(\mathrm{C}), 132.7(\mathrm{CH}), 131.3\left(\mathrm{~d},{ }^{4} J_{\mathrm{CF}}=2 \mathrm{~Hz}, \mathrm{C}\right), 130.3$ $(\mathrm{CH}), 130.1\left(\mathrm{~d},{ }^{3} J_{\mathrm{CF}}=8 \mathrm{~Hz}, \mathrm{C}\right), 117.1\left(\mathrm{~d},{ }^{2} J_{\mathrm{CF}}=24 \mathrm{~Hz}, \mathrm{CH}\right), 115.6(\mathrm{CH}), 114.4\left(\mathrm{~d},{ }^{2} J_{\mathrm{CF}}=23 \mathrm{~Hz}, \mathrm{CH}\right)$, $112.3\left(\mathrm{~d},{ }^{3} J_{\mathrm{CF}}=8 \mathrm{~Hz}, \mathrm{CH}\right), 109.9(\mathrm{C}), 56.2\left(\mathrm{CH}_{3}\right) ;{ }^{19} \mathrm{~F}-\mathrm{NMR} \delta\left(471 \mathrm{MHz}, \mathrm{CDCl}_{3}\right)-123.79(\mathrm{ddd}, J=9.2$, 8.3 and $4.5 \mathrm{~Hz}) ; \mathrm{m} / z$ (EI) $298\left({ }^{81} \mathrm{BrM}^{+}, 89 \%\right), 296\left({ }^{79} \mathrm{BrM}^{+}, 90\right), 203$ (20) 202 (100); HRMS (EI) ${ }^{79} \mathrm{BrM}^{+}$ found 295.9839, $\mathrm{C}_{13} \mathrm{H}_{10} \mathrm{O}_{2}{ }^{79} \mathrm{BrF}$ requires 295.9843 .

Methyl (S)-2-tert-butoxycarbonylamino-3-(3-bromo-4-hydroxyphenyl)propanoate (ent-6): $5.00 \mathrm{mmol} ; 1.68 \mathrm{~g}$ $(89 \%) ; \mathrm{Rt}=25.3 \mathrm{~min} ;[\alpha]_{\mathrm{D}}=59.0\left(\mathrm{c} 1, \mathrm{CHCl}_{3}\right) ; \mathrm{mp} 117-119{ }^{\circ} \mathrm{C} ;{ }^{1} \mathrm{H}-\mathrm{NMR} \delta\left(500 \mathrm{MHz}, \mathrm{CDCl}_{3}, 323 \mathrm{~K}\right)$ $7.24(1 \mathrm{H}, \mathrm{d}, J=2.0 \mathrm{~Hz}, \mathrm{ArH}), 6.97(1 \mathrm{H}, \mathrm{dd}, J=8.3$ and $2.0 \mathrm{~Hz}, \operatorname{ArH}), 6.90(1 \mathrm{H}, \mathrm{d}, J=8.3 \mathrm{~Hz}, \operatorname{ArH}), 5.50$ $\left(1 \mathrm{H}\right.$, br s, OH), $5.01(1 \mathrm{H}, \mathrm{br} \mathrm{s}, \mathrm{NH}), 4.51(1 \mathrm{H}, \mathrm{br} \mathrm{s}, \alpha-\mathrm{CH}), 3.71\left(3 \mathrm{H}, \mathrm{s}, \mathrm{OCH}_{3}\right), 3.04(1 \mathrm{H}, \mathrm{dd}, J=14.0$ and $\left.5.8 \mathrm{~Hz}, \mathrm{CH}_{\mathrm{A}} \mathrm{H}_{\mathrm{B}} \mathrm{Ar}\right), 2.97-2.88\left(1 \mathrm{H}, \mathrm{m}, \mathrm{CH}_{\mathrm{A}} \mathrm{H}_{\mathrm{B}} \mathrm{Ar}\right), 1.43\left(9 \mathrm{H}, \mathrm{s}, \mathrm{C}\left(\mathrm{CH}_{3}\right)_{3}\right) ;{ }^{13} \mathrm{C}-\mathrm{NMR} \delta(126 \mathrm{MHz}$, $\left.\mathrm{CDCl}_{3}, 323 \mathrm{~K}\right) 172.2(\mathrm{C}), 155.2(\mathrm{C}), 151.8(\mathrm{C}), 133.0(\mathrm{CH}), 130.1(\mathrm{CH}), 129.8(\mathrm{C}), 116.3(\mathrm{CH}), 110.2(\mathrm{C})$, $80.3(\mathrm{C}), 54.7(\mathrm{CH}), 52.3\left(\mathrm{CH}_{3}\right), 37.5\left(\mathrm{CH}_{2}\right), 28.4\left(3 \times \mathrm{CH}_{3}\right) ; m / z(\mathrm{ESI}+, \mathrm{MeOH}) 398\left([81 \mathrm{BrM}+\mathrm{Na}]^{+}\right.$, 100\%), 396 ([79BrM + Na $\left.{ }^{+}, 99\right), 374$ (14) 342 (10), 340 (11); HRMS (ESI+, MeOH) $[\mathrm{M}+\mathrm{Na}]^{+}$found 396.0434, $\mathrm{C}_{15} \mathrm{H}_{20} \mathrm{O}_{5} \mathrm{~N}^{79} \mathrm{BrNa}$ requires 396.0417.

\section{Conclusions}

In assessing the use of methanol as a carrier solvent for the mono ortho-bromination of phenols by NBS under flow conditions, the "dark reaction" clearly indicated that UV-vis irradiation was not a prerequisite for success. Thus batch reaction conditions were optimized to allow a range of NBS-mediated mono ortho-bromination reactions to be carried out in ACS-grade methanol on gram scale without UV-vis irradiation in reaction times of $25 \mathrm{~min}$. The highly selective product distributions achieved meant that the desired products of these reactions were readily purified in excellent yields $(86 \%-98 \%)$, thus providing an extremely facile route to high value medicinal chemistry building blocks. With an efficient route to the synthesis of the mono ortho-brominated derivative of Boc-D-Tyr-OMe in hand, the synthesis of the intriguing anti-cancer natural product, bisebromoamide, may now be tackled. 
Supplementary Materials: The following are available online at http://www.mdpi.com/1420-3049/21/1/88/s1, Figure S1: Choice of wavelength for reaction monitoring, Figure S2: ${ }^{1} \mathrm{H}$ - and ${ }^{13} \mathrm{C}-\mathrm{NMR}$ spectra for compounds 15 and ent-6.

Acknowledgments: We thank Cancer Research UK (Grant Reference C21383/A6950), EPSRC and BBSRC (studentship to SKB, BBS/S/H/2005/13535) for funding. We thank Alessio De Simone for the preparation of $3^{\prime}$-fluoro-6'-methoxy-[1,1'-biphenyl]-4-ol starting material.

Author Contributions: H.J.J., S.K.B. and A.H. designed and performed the experiments described in the introduction; D.G. and B.W.H.S. designed and performed the flow experiments; D.G. designed and performed the experiments in ACS-grade methanol; A.N.H. and D.G. wrote the paper.

Conflicts of Interest: The authors declare no conflict of interest.

\section{References}

1. Daum, S.; Erdmann, F.; Fischer, G.; Féaux-de-Lacroix, B.; Hessamian-Alinejad, A.; Houben, S.; Frank, W.; Braun, M. Aryl indanyl ketones: Efficient inhibitors of the human peptidyl prolyl cis/trans isomerase Pin1. Angew. Chem. Int. Ed. 2006, 45, 7454-7458. [CrossRef] [PubMed]

2. Crosignani, S.; Prêtre, A.; Jorand-Lebrun, C.; Fraboulet, G.; Seenisamy, J.; Augustine, J.K.; Missotten, M.; Humbert, Y.; Cleva, C.; Abla, N.; et al. Discovery of potent, selective, and orally bioavailable alkynylphenoxyacetic acid CRTH2 (DP2) receptor antagonists for the treatment of allergic inflammatory diseases. J. Med. Chem. 2011, 54, 7299-7317. [CrossRef] [PubMed]

3. Teruya, T.; Sasaki, H.; Fukazawa, H.; Suenaga, K. Bisebromoamide, a potent cytotoxic peptide from the marine cyanobacterium Lyngbya sp.: Isolation, stereostructure, and biological activity. Org. Lett. 2009, 11, 5062-5065. [CrossRef] [PubMed]

4. Gao, X.G.; Liu, Y.Q.; Kwong, S.Q.; Xu, Z.S.; Ye, T. Total synthesis and stereochemical reassignment of bisebromoamide. Org. Lett. 2010, 12, 3018-3021. [CrossRef] [PubMed]

5. Li, W.H.; Yu, S.Y.; Jin, M.Z.; Xia, H.G.; Ma, D.W. Total synthesis and cytotoxicity of bisebromoamide and its analogues. Tetrahedron Lett. 2011, 52, 2124-2127. [CrossRef]

6. Boys, S.K. Tyrosine Derivatives and Their Anti-Cancer Applications. Ph.D. Thesis, The University of Edinburgh, Edinburgh, UK, 2012.

7. Johnston, H.J. Development of Novel Analogues of the Anti-Proliferative Marine Natural Product Bisebromoamide: Synthesis and Structure Activity Relationship Studies. Ph.D. Thesis, The University of Edinburgh, Edinburgh, UK, 2014.

8. Johnston, H.J.; McWhinnie, F.S.; Landi, F.; Hulme, A.N. Flexible, phase-transfer catalyzed approaches to 4-substituted prolines. Org. Lett. 2014, 16, 4778-4781. [CrossRef] [PubMed]

9. Johnston, H.J.; Hulme, A.N. A facile, inexpensive and scalable route to thiol-protected $\alpha$-methyl cysteine. Synlett 2013, 24, 591-594.

10. Zhou, C.-Y.; Li, J.; Peddibhotla, S.; Romo, D. Mild arming and derivatization of natural products via an In(OTf $)_{3}$-catalyzed arene iodination. Org. Lett. 2010, 12, 2104-2107. [CrossRef] [PubMed]

11. Chhattise, P.K.; Ramaswamy, A.V.; Waghmode, S.B. Regioselective, photochemical bromination of aromatic compounds using N-bromosuccinimide. Tetrahedron Lett. 2008, 49, 189-194. [CrossRef]

12. Bovonsombat, P.; Khanthapura, P.; Krause, M.M.; Leykajarakul, J. Facile syntheses of 3-halo and mixed 3,5-dihalo analogues of $N$-acetyl-L-tyrosine via sulfonic acid-catalysed regioselective monohalogenation. Tetrahedron Lett. 2008, 49, 7008-7011. [CrossRef]

13. Bovonsombat, P.; Ali, R.; Khan, C.; Leykajarakul, J.; Pla-on, K.; Aphimanchindakul, S.; Pungcharoenpong, N.; Timsuea, N.; Arunrat, A.; Punpongjareorn, N. Facile $p$-toluenesulfonic acid-promoted para-selective monobromination and chlorination of phenol and analogues. Tetrahedron 2010, 66, 6928-6935. [CrossRef]

14. Verner, E.; Katz, B.A.; Spencer, J.R.; Allen, D.; Hataye, J.; Hruzewicz, W.; Hui, H.C.; Kolesnikov, A.; Li, Y.; Luong, C.; et al. Development of serine protease inhibitors displaying a multicentered short $(<2.3 \AA)$ hydrogen bond binding mode: Inhibitors of urokinase-type plasminogen activator and factor Xa. J. Med. Chem. 2001, 44, 2753-2771.

15. Hartman, R.L.; McMullen, J.P.; Jensen, K.F. Deciding whether to go with the flow: Evaluating the merits of flow reactors for synthesis. Angew. Chem. Int. Ed. 2011, 50, 7502-7519. [CrossRef] [PubMed]

16. Lévesque, F.; Seeberger, P.H. Highly efficient continuous flow reactions using singlet oxygen as a "green" reagent. Org. Lett. 2011, 13, 5008-5011. [CrossRef] [PubMed] 
17. Knowles, J.P.; Elliott, L.D.; Booker-Milburn, K.I. Flow photochemistry: Old light through new windows. Beilstein J. Org. Chem. 2012, 8, 2025-2052. [CrossRef] [PubMed]

18. Wojcik, F.; O’Brien, A.G.; Götze, S.; Seeberger, P.H.; Hartmann, L. Synthesis of carbohydrate-functionalised sequence-defined oligo(amidoamine)s by photochemical thiol-ene coupling in a continuous flow reactor. Chem. Eur. J. 2013, 19, 3090-3098. [CrossRef] [PubMed]

19. McQuade, D.T.; Seeberger, P.H. Applying flow chemistry: Methods, materials, and multistep synthesis. J. Org. Chem. 2013, 78, 6384-6389. [CrossRef] [PubMed]

20. Bovonsombat, P.; McNelis, E. Ring halogenations of polyalkylbenzenes with $N$-halosuccinimide and acid catalysts. Synthesis 1993, 237-241. [CrossRef]

21. Mahajan, T.; Kumar, L.; Dwivedi, K.; Agarwal, D.D. Efficient and facile chlorination of industrially-important aromatic compounds using $\mathrm{NaCl} / p-\mathrm{TsOH} / \mathrm{NCS}$ in aqueous media. Ind. Eng. Chem. Res. 2012, 51, 3881-3886. [CrossRef]

22. Stock, R.I.; Nandi, L.G.; Nicoleti, C.R.; Schramm, A.D.S.; Meller, S.L.; Heying, R.S.; Coimbra, D.F.; Andriani, K.F.; Caramori, G.F.; Bortoluzzi, A.J.; et al. Synthesis and solvatochromism of substituted 4-(nitrostyryl)phenolate dyes. J. Org. Chem. 2015, 80, 7971-7983. [CrossRef] [PubMed]

23. Mangas-Sánchez, J.; Busto, E.; Gotor-Fernández, V.; Gotor, V. Straightforward synthesis of enantiopure 2,3-dihydrobenzofurans by a sequential stereoselective biotransformation and chemical intramolecular cyclization. Org. Lett. 2010, 12, 3498-3501. [CrossRef] [PubMed]

24. Adimurthy, S.; Ramachandraiah, G.; Bedekar, A.V.; Ghosh, S.; Ranu, B.C.; Ghosh, P.K. Eco-friendly and versatile brominating reagent prepared from a liquid bromine precursor. Green Chem. 2006, 8, $916-922$. [CrossRef]

25. Huang, G.-J.; Bhaskar-Reddy, M.V.; Kuo, P.-C.; Huang, C.-H.; Shih, H.-C.; Lee, E.J.; Yang, M.-L.; Leu, Y.-L.; $\mathrm{Wu}, \mathrm{T}$.-S. A Concise synthesis of viscolin, and its anti-inflammatory effects through the suppression of iNOS, COX-2, ERK phosphorylation and proinflammatory cytokines expressions. Eur. J. Med. Chem. 2012, 48, 371-378. [CrossRef] [PubMed]

26. Podgoršek, A.; Stavber, S.; Zupan, M.; Iskra, J. Environmentally benign electrophilic and radical bromination "on water": $\mathrm{H}_{2} \mathrm{O}_{2}-\mathrm{HBr}$ system versus $N$-bromosuccinimide. Tetrahedron 2009, 65, 4429-4439. [CrossRef]

27. Schmidt, B.; Holter, F. Suzuki-Miyaura cross coupling reactions with phenoldiazonium salts. Org. Biomol. Chem. 2011, 9, 4914-4920. [CrossRef] [PubMed]

Sample Availability: Samples of the compounds are available from the authors.

(C) 2016 by the authors; licensee MDPI, Basel, Switzerland. This article is an open access article distributed under the terms and conditions of the Creative Commons by Attribution (CC-BY) license (http:/ / creativecommons.org/licenses/by/4.0/). 